\title{
AN AUTONOMOUS ROBOT EQUIPPED WITH THE GPS VIRTUAL REFERENCE STATION (VRS) SYSTEM TO PERFORM PAVEMENT DISTRESS SURVEYS
}

\author{
Jia-Ruey Chang \\ Associate Professor \\ Department of Civil Engineering \\ MingHsin University of Science \& Technology \\ No. 1, Hsin-Hsing Road, Hsin-Chu 304, Taiwan \\ (T) 886-3-5593142-3295; (F) 886-3-5573718 \\ jrchang@must.edu.tw \\ Peter M. Liu \\ Postdoctoral Researcher \\ Department of Civil Engineering \\ National Taiwan University \\ No. 1, Sec. 4, Roosevelt Road, Taipei 106, Taiwan \\ (T) 886-2-33664346; (F) 886-2-2368-8213 \\ r96749009@ntu.edu.tw

\section{Tsun-Cheng Huang} \\ Master Student \\ Department of Civil Engineering \\ MingHsin University of Science \& Technology \\ No. 1, Hsin-Hsing Road, Hsin-Chu 304, Taiwan \\ (T) 886-3-5593142-3281; (F) 886-3-5573718 \\ chenww5828@yahoo.com.tw
}

\author{
Shih-Chung Kang \\ Assistant Professor \\ Department of Civil Engineering \\ National Taiwan University
}

No. 1, Sec. 4, Roosevelt Road, Taipei 106, Taiwan

(T) 886-2-33664346; (F) 886-2-2368-8213

sckang@caece.net

Shang-Hsien Hsieh

Professor

Department of Civil Engineering

National Taiwan University

No. 1, Sec. 4, Roosevelt Road, Taipei 106, Taiwan

(T) 886-3-2-33664313; (F) 886-2-2368-8213

shhsieh@caece.net

Ping-Hung Lin

Master Student

Department of Civil Engineering

National Taiwan University

No. 1, Sec. 4, Roosevelt Road, Taipei 106, Taiwan

(T) 886-2-33664346; (F) 886-2-2368-8213

b92501030@ntu.edu.tw

\begin{abstract}
Various pavement distresses (cracking, pothole, manhole, patching, etc.) that exist randomly across pavements deteriorate the quality of the pavement structure and need to be immediately dealt using proper maintenance and rehabilitation (M\&R) strategies. Traditionally pavement distress surveys are performed using manually operated or driven equipment, and hence are very labour-intensive, time-consuming and lack intelligent detection methods. In this study, an autonomous robot platform was pioneered to conduct pavement distress surveys. For accurate data collection and robot mobility on broad pavement, a positioning system with high accuracy and a short initialization time is needed. The main objective of this
\end{abstract}


study is to integrate Leica's GPS VRS system with a P3-AT robot to conduct pavement distress surveys effectively. Pavement distresses can be located to the nearest centimeter. Through field tests, the feasibility of this architecture was examined.

\section{KEYWORDS}

Pavement distress survey, Autonomous robot, Global positioning system (GPS), Virtual reference station (VRS) system

\section{INTRODUCTION}

Pavement-management decisions are factor in the methods of evaluating pavement conditions, predict future performance, rank the severity of problems, prioritize needs, and determine the required repair levels. Current and accurate distress data (including severity, coverage, and positioning of each distress) are needed to document the present condition of the pavement, to determine maintenance and rehabilitation (M\&R) needs, and to form the basis of a pavement management system. Traditionally, pavement distress surveys are performed using a repetitive and time-consuming procedure; it uses humanoperated or driven equipment, and hence are very labour-intensive, time-consuming and lack intelligent detection methods. It is very difficult to collect pavement distress data safely, accurately, and intelligently on broad pavements. To solve these problems, this study pioneered the application of an autonomous robot equipped with a high-accuracy positioning system to pavement distress surveying by exploiting robots' features of mobility, precision, and autonomy from human control to save on time and cost. The robot is able to search for pavement distresses, capture distress images, and plan the upcoming motion in real-time. This allows engineers to collect pavement distress data in a more effective way.

Global Positioning System (GPS) Real-Time Kinematic (RTK) positioning is becoming increasingly important for many precise GPS applications, with examples including surveying, construction, precision farming and high accuracy Geographic Information Systems (GIS). Traditionally, a user receiver requires a reference station within $10 \mathrm{~km}$ to ensure centimeter-level accuracy. Recently, multiple reference station networks have been installed in many countries to overcome the limitations of standard RTK systems. The concept of the Virtual Reference Station (VRS) is one of the more feasible approaches for relaying network correction information to the network RTK users. For accurately positioning the robot on pavements, a positioning system with high accuracy (centimeterlevel), Leica's GPS VRS system, was equipped on the P3-AT autonomous robot. In this study, a demonstration was performed on an in-situ pavement to assess the feasibility of this architecture for distress data collection.

\section{GPS VIRTUAL REFERENCE STATION (VRS) SYSTEM}

RTK positioning in conjunction with GPS can reach a high accuracy (to the centimeter-level) and is one of the most widely used surveying techniques today. But, RTK has some drawbacks such as the effects of the troposphere and ionosphere which result in systematic errors in raw data; the distance between a rover (mobile) receiver and its reference (base) station has to be quite short (less than $10 \mathrm{~km}$ ) in order to work efficiently. Also radio communication problems arise in urban areas.

Recently, the use of the VRS, so-called network RTK, concept has been proposed by many researchers as a more feasible approach for relaying network correction information to the network RTK users [1-5] and has proven an efficient technology for high accuracy GPS positioning over the last few years. This approach does not require an actual physical reference station. Instead, it allows the user to access data of a non-existent virtual reference station at any location within the network coverage area and allows modelling the systematic errors to provide the possibility of an error reduction. VRS network operation is shown in Figure 1 [6]. The advantages of the VRS technique follow.

- increase the allowable distance between the rover receiver and the reference station;

- remove large portions of ionospheric and geometric errors through network corrections; 


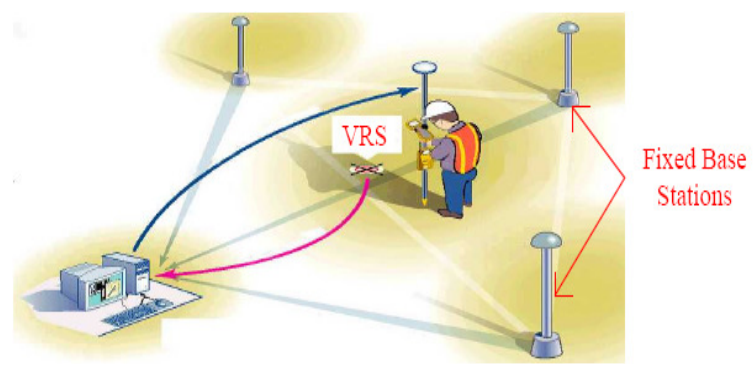

Figure 1. VRS Network Operation [6]

- increase the reliability and productivity of ambiguity resolution and the positioning accuracy of rovers;

- reduce the initialization time of RTK.

The VRS approach is flexible: users can utilize their current receivers and software without any specific software to manage corrections from a series of reference stations. Users within the reference station network can operate reliably at greater distances without a degradation in accuracy. However, it is necessary to provide a reliable data communication link for the transmission of VRS data from a control centre to a user receiver [7]. There are several practical approaches to transfer the VRS data to users in real-time, such as TV audio data broadcast, GSM, Internet, etc [3, 8]. In this study, a General Packet Radio Service (GPRS) is utilized as the data transmission device to position the robot on pavements. GPRS provides an "always-on" service, and is thus preferable to GSM. It provides a stable and reliable connection with latencies of less than one second. Figure 2 shows the architecture for linking the control centre with the user receiver using GPRS [7]. The PDA in Figure 2 is replaced with an onboard laptop on the autonomous robot in this study.

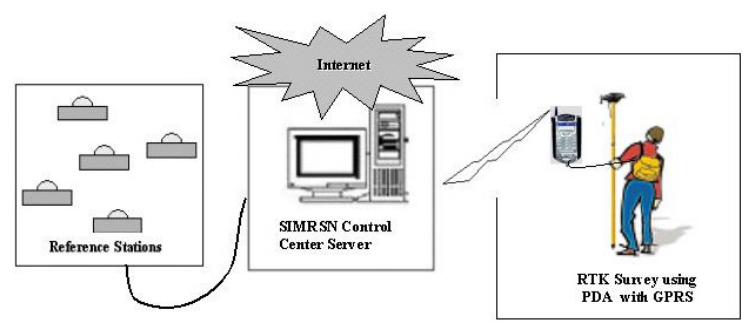

Figure 2. The Architecture of Internet-based VRS System via GPRS [7]

\section{AN AUTONOMOUS ROBOT: MOBILEROBOTS P3-AT}

Being powerful, easy-to-use, reliable, and flexible, the Pioneer 3-AT (P3-AT) used in this study is a highly versatile all-terrain robotic platform particularly suited for pavement inspections. Figure 3 shows the P3-AT.

\subsubsection{Introduction to P3-AT}

The P3-AT is manufactured by MobileRobots Inc. and is equipped with a control laptop, onboard PanTilt-Zoom (PTZ) camera system, Ethernet-based communications, SICK laser, eight forward and eight rear sonars which sense obstacles from $15 \mathrm{~cm}$ to $7 \mathrm{~m}$, and other autonomous functions. P3-AT's powerful motors and four robust wheels can reach speeds of $0.8 \mathrm{~m} / \mathrm{sec}$ and carry a payload of up to 30 $\mathrm{kg}$. P3-AT can climb steep $45 \%$ grades and sills of 9 cm. P3-AT uses 100-tick encoders with inertial correction recommended for dead reckoning to compensate for skid steering. Its sensing extends far beyond the ordinary with laser-based navigation options, integrated inertial correction to compensate for slippage, bumpers, gripper, vision, stereo rangefinders, compass and a rapidly growing suite of other options [9].

The bare P3-AT base with included Advanced Robotics Interface Application (ARIA) software has the ability to [9]:

- $\quad$ wander randomly on pavements;

- drive controlled by keys or joystick;

- plan survey paths with gradient navigation on pavements;

- display a pavement spatial map of its sonar and/or laser readings;

- localize using sonar or laser upgrade;

- communicate sensor and control information relating sonar, motor encoder, motor controls, user I/O, and battery charge data;

- test pavement survey activities quickly with ARIA API from $\mathrm{C}^{++}$programs;

- simulate pavement survey behaviours offline with the simulator that accompanies each development environment. 


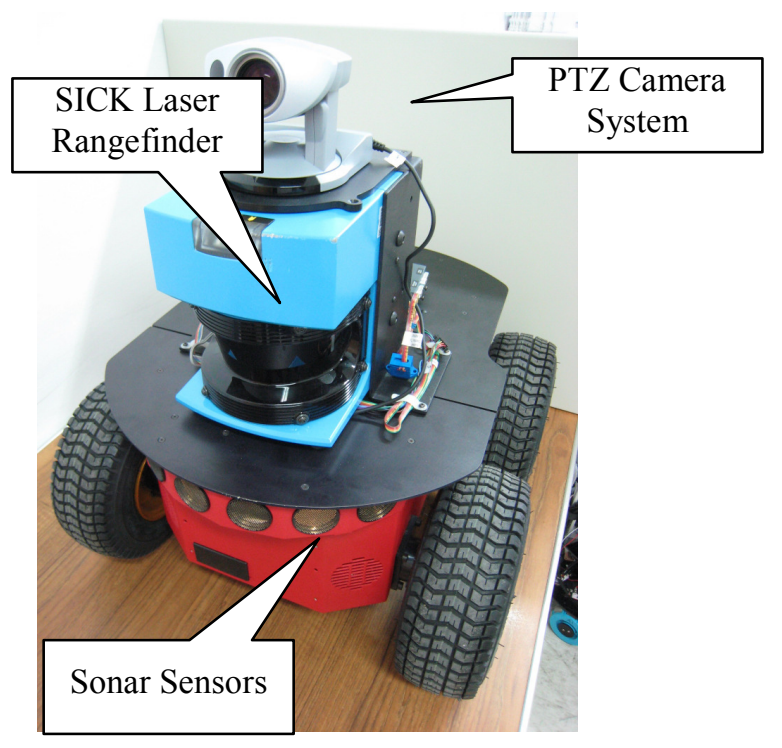

Figure 3. MobileRobots P3-AT

\subsubsection{P3-AT Vision System}

The Pan-Tilt-Zoom (PTZ) system on the P3-AT provides an integrated robotic camera whose movement can be easily controlled via ARIA development software. This camera can be configured for pavement distress survey purposes with specific vision routines [10].

The PTZ robotic camera system is fully integrated with ARCOS (robot hardware and operating system software) and ARIA software plug-ins for easy operation. The system includes:

- 26 times (at infinite) optical, 12X digital zoom for wide range of applications;

- high-speed auto-focus lens for responsiveness;

- $\quad+/-100$ degree pan at up to 90 degrees/sec;

- $\quad-30$ to +90 degree tilt at up to 70 degrees/sec;

- connections to plug into MobileRobots microcontroller RS232C serial port; and,

- all NTSC functions.

ARCOS and ARIA command sets include:

- $\quad$ pan/tilt to $\mathrm{x} / \mathrm{y}$ degrees (absolute position);

- $\quad$ pan/tilt x/y degrees (relative position);

- $1 \mathrm{X}-26 \mathrm{X}$ zoom.

\section{GPS VRS SYSTEM INTRGRATED WITH P3-AT TO PAVEMENT DISTRESS SURVEYS}

A pavement distress survey was conducted to monitor the present pavement condition and determine the appropriate $M \& R$ activities. The common distress types include alligator cracking, longitudinal cracking, transverse cracking, block cracking, rutting, and patching, etc. Toward the end of the 1970s, Darter and Shahin [11] developed the Pavement Condition Index (PCI), which has been referred to as ASTM D6433 [12] for roads and parking Lots and ASTM D5340 [13] for airfield pavement. Manual and automatic approaches are the two major ways, adopted all over the world, to perform a pavement distress survey. The manual approach is conducted through visual observation. The severity, coverage, and positioning of each distress on one pavement unit are identified and recorded using pen and paper or a personal digital assistant (PDA) equipped with GPS. The PCIs can be computed immediately using the PDA or manually during post-processing. The automatic approach is to first capture pavement images with GPS information, and then the severity and coverage of each distress are digitally identified using image processing algorithms. However, present image processing merely focuses on linear cracking and potholes thus could not replace the manual approach. Significant developments have taken place in automating distress measurement. There are many inertial profilers that automate the complete distress data collection. More literature can be found at [14, $15]$.

Most distress surveys on broad pavements are performed using a repetitive and time-consuming procedure that uses human-operated instruments, hence are very labour-intensive, time-consuming and lack the application of intelligent technologies. To solve these problems, this study proposes the idea of applying P3-AT equipped with an Internetbased VRS system via GPRS to pavement distress surveys by utilizing the robot's features of mobility, high accuracy, and time and labour efficiencies. P3AT will search pavement distresses, capture distress images with positioning information in real time, and plan the upcoming motion in real-time. This 
helps us collect pavement distress data in a more effective way.

\subsubsection{GPS VRS System Operation}

Software, SpiderNET, at the control-centre server located at the Control Signal Company Limited has been written to generate and broadcast VRS data via the Internet. The software opens the ports and waits for the P3-AT connection. There is a login procedure required to gain access to the data. The software handles incoming logins and generates the VRS data for P3-AT according to P3-AT's approximate position.

On the robot side, P3-AT is equipped with a Leica SR530 receiver (Figure 4) capable of performing RTK positioning and is connected to a GPRSenabled laptop via the RS232 port (COM 1). Client software, Ntrip Client, was installed on the PC environment, as shown in Figure 5. After filling the IP port, User-ID, and password in the Ntrip Client, the laptop establishes communication with the receiver through another RS232 port (COM 2), as shown in Figure 6. P3-AT can connect to the server of the control centre through GPRS at its location. The positioning quality can reach to within $0.01 \mathrm{~m}$ (centimeter-level), as shown in Figure 7.

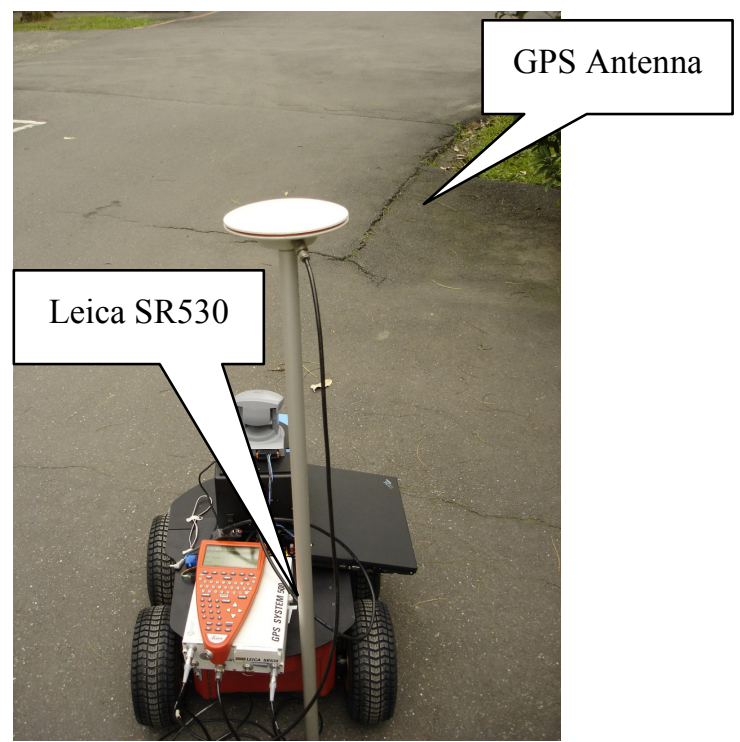

Figure 4. The Profile of P3-AT for Pavement Distress Surveys

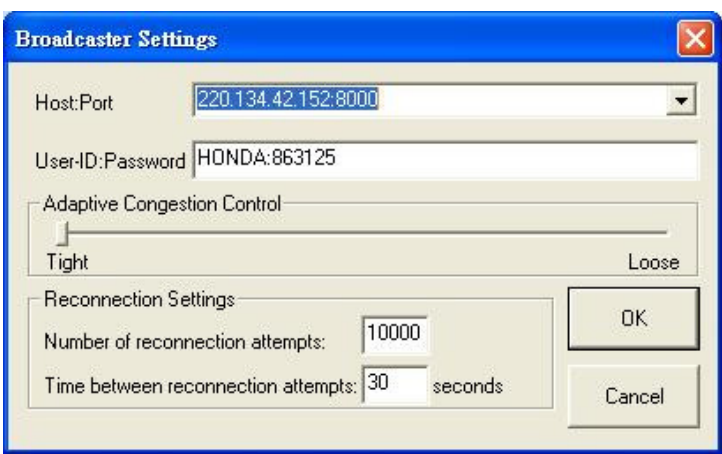

Figure 5. Ntrip Client Software

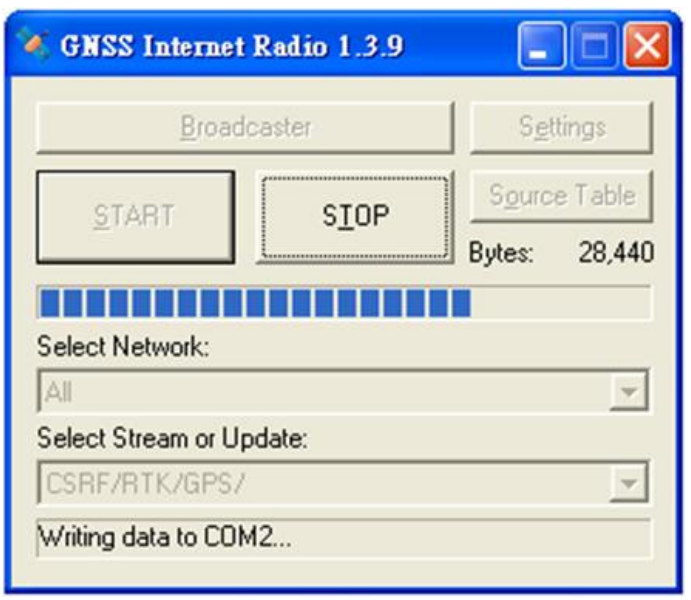

Figure 6. VRS Data Access by Ntrip Client

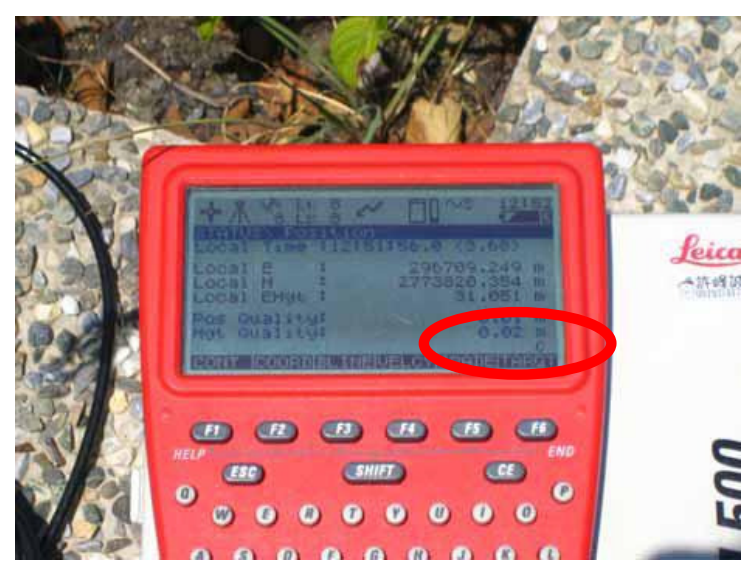

Figure 7. P3-AT Positioning Quality Reaches to $0.01 \mathrm{~m}$ (centimeter-level) 


\subsubsection{Pavement Distress Surveys}

The distress images were automatically captured by the onboard PTZ camera system and recorded with positioning information of centimeter-level quality in real-time, as shown in Figure 8. The image shown in Figure 8 was captured at 07:44:55am in 2008-323. The cracking on the pavement occurs at North Latitude 2501.1342162 (WGS 1984) and East Longitude 12132.3516293 (WGS 1984), according to the data superimposed on the image. However, at present, the distress recognition and PCIs computation were conducted manually during the post-processing. Development of an overall automatic process is ongoing.

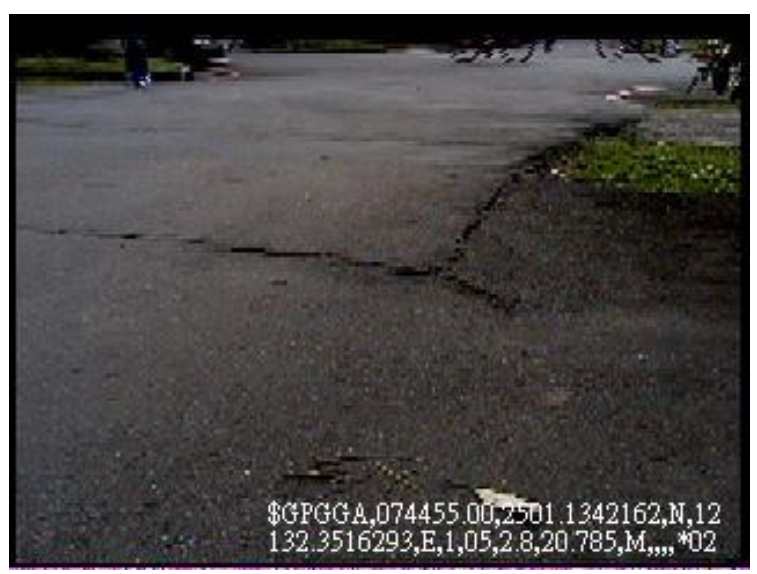

Figure 8. A Cracking Image Captured by P3-AT with Positioning Information (centimeter-level)

\section{CONCLUSIONS}

For correct pavement data collection and mobility of a robot on broad pavements, a positioning system that possesses high accuracy, allows for a longer distance between the GPS receiver and the reference station and is also free of radio communication problems in urban areas is required. The Internetbased VRS system is a real-time, centimeter-level service for construction, rapid surveying, and GIS.

In the study, Leica's GPS VRS system is integrated with an autonomous robot P3-AT and applied successfully in the pavement distress surveys for high accuracy positioning acquisition via GPRS. It can also be used in related pavement inspection applications including inspections for longitudinal evenness (roughness) and transversal evenness (rutting). This helps us to collect pavement condition data in a more effective way.

\section{ACKNOWLEDGEMENTS}

7.The authors would like to thank the National Science Council of Taiwan for their financial support provided under the project of NSC 96-2628E-159-002-MY3, and the technical support received from the Control Signal Company Limited in Taiwan.

\section{REFERENCES}

[1] Wanninger, L. (1997) Real-time differential GPSerror modelling in regional reference station networks, Proc. of the IAG Scientific Assembly, Rio de Janeiro, September, IAG-Symposia 118, Springer Verlag, 86-92.

[2] Marel, H. (1998) Virtual GPS reference stations in the Netherlands, Proc. 11th Int. Tech. Meeting of the Satellite Division of the U.S. Inst. of Navigation, ION GPS-98, Nashville, TN, September 15-18, 4958.

[3] Vollath, U., Buecherl, A., Landau, H., Pagels, C., \& Wager, B. (2000) Multi-base RTK positioning using virtual reference stations, Proc. 13th Int. Tech. Meeting of the Satellite Division of the U.S. Inst. of Navigation, ION GPS-2000, Salt Lake City, September 19-22, 123-131.

[4] Cannon, M.E., Lachapelle, G., Fortes, L.P., Alves, P., \& Townsend, B. (2001) The use of multiple reference station VRS for precise kinematic positioning, Proc. of the Japan Institute of Navigation, GPS Symposium 2001, Tokyo, November 14-16, 29-37.

[5] Euler, H.J., Keenan, C.R., Zebhauser, B.E., \& Wübbena, G. (2001) Study of a simplified approach in utilizing information from permanent reference station arrays, Proc. 14th International Technical Meeting of the Satellite Division of the Institute of Navigation, Salt Lake City, USA, ION GPS-2001, September 11-14, 379-391.

[6] Trimble Virtual Reference Station $\left(\mathrm{VRS}^{\mathrm{TM}}\right)$ System (2005), Versatile RTK positioning infrastructure for local and regional applications, Retrieved March 10, 2008, from http://trl.trimble.com/docushare/dsweb/ Get/Document-11465/VRS_Spec_Sheet.pdf

[7] Hu, G.R., Khoo, V.H.S., Goh, P.C., \& Law, C.L. (2002) Internet-based GPS VRS RTK Positioning with a Multiple Reference Station Network, Journal 
of Global Positioning Systems, Vol. 1, No. 2, 113120.

[8] Liu, Z. \& Gao, Y. (2001) Research toward wireless Internet-based DGPS, Proceedings of KIS 2001, Banff, Canada, June 5-8, 461-469.

[9] MobileRobots Inc. (2008) The high performance allterrain robot - P3-AT, Retrieved January 21, 2008,

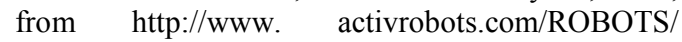
p2at.html

[10] MobileRobots Inc. (2008) Robotic Pan-Tilt-Zoom camera systems, Retrieved Feb. 13, 2008, from http://www.activrobots.com/ACCESSORIES/ ptzvideo.html\#robocam

[11] Darter, M.I. \& Shahin, M.Y. (1980) Pavement rehabilitation: Identifying the need, Journal of Transportation Engineering, Vol. 106, No. 1, 1-10.

[12] ASTM D6433 (2003) Standard Practice of Roads and Parking Lots Pavement Condition Index Surveys.

[13] ASTM D5340 (2004) Standard Test Method for Airport Pavement Condition Index Surveys.

[14] Wang, K.C.P. (2003) Transportation Research Circular: Automated Imaging Technologies for Pavement Distress Survey, Committee A2B06, Transportation Research Board, National Research Council, Washington, D.C.

[15] Wang, K.C.P. \& Li, X. (1999) Use of digital camera for pavement surface distress survey, Transportation Research Record 1675, Transportation Research Board, National Research Council, Washington, D.C., 91-97. 